\title{
Modification of Passivation Unit in Continuous Galvanizing Line
}

\author{
S.P.Zadake ${ }^{t^{*}}$ and .S.G.Dambhare ${ }^{\ddagger}$ \\ †Heat Power Engineering, S.P. Pune University, Pune. Maharashtra, India \\ ^Mechanical Engineering, S.P. Pune University, Pune. Maharashtra, India \\ Accepted 15 June 2016, Available online 20 June 2016, Special Issue-5 (June 2016)
}

\begin{abstract}
Galvanizing is the process of giving a protective zinc coating to steel or iron, to avoid rusting. The most common method is hot-dip galvanizing, in which steel in coil form is dipped in a bath of molten zinc. Galvanizing protects in two ways:-It generates a coating of corrosion-resistant zinc which prevents corrosive substances from reaching the more delicate part of the metal and increase the life of steel. The zinc serves as protecting layer so that even if the coating is removed by scratching, the exposed steel will still be protected by the remaining zinc coating. Hot-dip galvanizing deposits a thick rough zinc layer that may be excessive. In the case of automobile bodies, where additional glossy paint will be applied, a thinner form of galvanizing is applied by electro galvanization. The hot-dip process changes the mechanical properties of the material by doing continuous annealing in furnace. For cleaning mixture hydrogen and nitrogen is preferred. This is a consideration for the processer of wire rope and other highly stressed products. The zinc layer provided by hot dip galvanizing is insufficient for products that will be constantly exposed to corrosive materials such as moisture. For these applications, more expensive stainless steel is preferred. Some nails made today are electro-galvanized. Nonetheless, powder coating is used on its own for many outdoor applications because it is cheaper than hot dip zinc coating and looks good when new but time taking process. The project work is on Passivation unit of hot dip galvanizing line. In this project problems occurred in passivation are rectified.
\end{abstract}

Keywords: Passivation, hot air generator, blower, duct, Pareto analysis

\section{Introduction}

Passivation in continuous galvanizing line refers to a material becoming passive, that is, being less affected by environmental factors such as air and moisture. Passivation involves a shielding outer-layer of base material, which can be applied as a zinc coating, or oxidation which occurs spontaneously in nature.

Passivation is required for galvanized steel to increase storage life, to prevent from white rust. For passivation there are two types of solutions are used one is chromic acid hexavalent and another is chromic acid trivalent. Chromic acid (hexavalent) is used for industrial use and chromic acid (trivalent) is used for home appliance galvanized steel. The project work based on problems occurred in Passivation which create defect on galvanized steel strip. The work of project is under progress in Uttam Galva Steels Limited, Khopoli. Problems occurred in Passivation unit are

1) Deposition of Passivation solution on deflector roll which after drying unit of Passivation
2) Moisture accumulated in Dryer unit.

3) Powder formation of solution due to improper temperature in drying unit.

4) Dent generation on finish product

5) Processed material loss due to above problems

6) Loss of productivity

\section{Passivation Process Diagram}

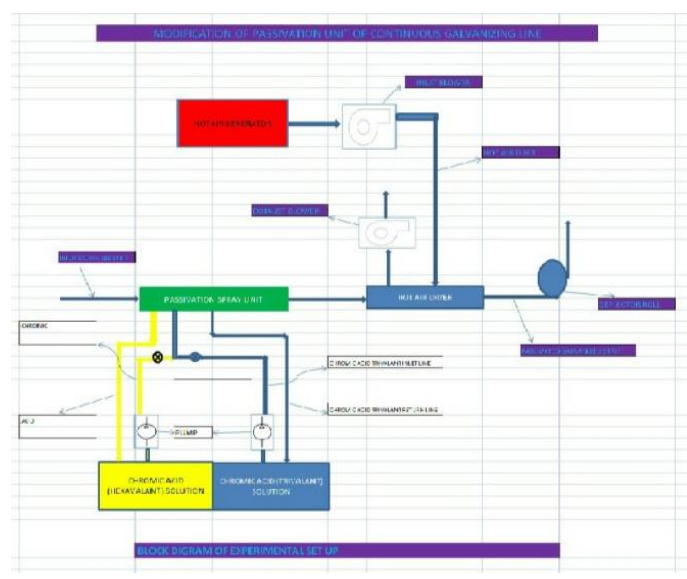

Fig.1 Passivation process diagram on line 
Following equipments comes under the Passivation unit:

1) Passivation solution tank

2) Spray pump

3) Hot air generator

4) Hot air carrying duct

5) Hot air dyer unit

6) Blower

7) Exhaust blower

\subsection{Problem Statement:}

Problems occurred in Passivation unit are

1) Deposition of Passivation solution on deflector roll which after drying unit of Passivation

2) Moisture accumulated in Dryer unit.

3) Powder formation of solution due to improper temperature in drying unit.

4) Dent generation on finish product

5) Processed material loss due to above problems

6) Loss of productivity.

To resolve this problem is project agenda to run the passivation unit smoothly and with full of its capacity.

\subsection{Dimensions of hot air dryer}

In passivation unit first solution is sprayed of the running strip and it goes in hot air dryer for drying purpose so we need to consider the dimensions' of the hot air dryer for calculation.

Length of the hot air dryer $=5 \mathrm{~m}$.

Width of the dryer $=1.6 \mathrm{~m}$

$$
\text { Drying.time }=\frac{\text { dryer.length }}{\text { process.speed }}
$$

\section{Literature Review}

Hong Wang Characterization of Dark Green Passivation Film On Galvanized Steel Sheet College of Mechanical and Electronic Engineering, Weifang University, Weifang, China Received: October 17, 2013 The blackishgreen Passivation handicraft we designed just has good compatibility with the potassium chloride zinc plating handicraft one of the green environmental protection electroplating produces handicraft. The appearance of galvanized sample treated by this Passivation craft was blackish green, colour was uniform and lustre was gentle. The Passivation film can endure neutral salt spray test for $400 \mathrm{~h}$. The passive films were characterized in this paper to determine its microstructure, phase composition and corrosion resistance.

Jan R. van der Net The Way Forward in Passivation of Galvanized Steel Coils Pauline Kleijn, Wilma van Leeuwen, Thomas Werner, Quaker Chemical B.V., Uithoorn, The Netherlands MPT International 6/2012 The performance of Quaker's next generation Cr (III) systems represents a leap in the performance of non- toxic Passivation systems. Thinner, more compact layers offer better corrosion protection than conventional $\mathrm{Cr}$ (III) or $\mathrm{Cr}$-free products. Also adhesion performance proved to be high in tests with glues and paints and a wide range of layer thicknesses. The Quaker PrimecoatTM is applicable with standard Passivation equipment available in galvanizing lines. Specific enhanced drying conditions are not required. A transparent surface finish is achieved with no discoloration even at higher layer thicknesses. Nontoxic ingredients - i.e. fluoride-free solution and absence of heavy metals - reduce both toxicity and the risk of etching when being applied to common stainless steel qualities. Finally, an excellent cost-benefit ratio compared to other Passivation products makes them a favourable choice for hot dip galvanizing

T.S.N. Sankara NarayananSurface Pretreatment by Phosphate Conversion Coatings A Review National Metallurgical Laboratory, Madras Centre CSIR, Complex, Taramani, and Chennai-600 113, India

After chromic acid rinsing the parts must be dried before finishing, the conventional methods used being simple evaporation, forced drying by blowing air or by heating [88]. Where evaporation conditions are good, warm air circulating fans and compressed air blow offs are the most economical methods. After drying the phosphate panels are ready for application of further finishes such as paints, oils, varnishes, etc.

Virendra V. Khakre, Avinash M. Wankhade Review paper on Design and Computational Analysis of Air Flow through Cooling Duct International Journal of Scientific \& Engineering Research, Volume 5, Issue 9, September-2014

The calculated value of frictional pressure drop is less as compared to existing plant or value used in industry. Due to less value, duct diameter is increased but loss in static pressure, velocity pressure can be avoided. Smaller diameter of duct would increase noise level. So requirement of sound attenuating devices may need. Also probability of dampers is decreased with increasing diameter. But first cost is increased with increasing duct diameter. Due to proper branching (with elbow) of ducts, loss is minimized in this design. But in existing plant, there is straight branching in so many locations, which may increase the pressure loss. Pressure loss in duct fitting is kept minimum by using elbow with proper shape considering very less pressure loss coefficient

M.Sampathkumar, Dsvsra Varaprasad, Vijaykumar, Static Analysis of Centrifugal Blower Using Composite Material The International Journal Of Engineering And Science (IJES), 2014, Centrifugal blowers look more like centrifugal pumps than fans. The impeller is typically gear-driven and rotates as fast as 15,000 rpm. In multi-stage blowers, air is accelerated as it passes through each impeller. In single-stage blower, air does not take many turns, and hence it is more efficient. Centrifugal blowers typically operate against pressures of 0.35 to $0.70 \mathrm{~kg} / \mathrm{cm} 2$, but can achieve higher pressures. One characteristic is that airflow tends to 
drop drastically as system pressure increases, which can be a disadvantage in material conveying systems that depend on a steady air volume. Because of this, they are most often used in applications that are not prone to clogging. Most manufacturing plants use fans and blowers for ventilation and for industrial processes that need an air flow. Fan systems are essential to keep manufacturing processes working, and consist of a fan, an electric motor, a drive system, ducts or piping, flow control devices, and air conditioning equipment (filters, cooling coils, heat exchangers, etc.).

\section{Variables of the Project Work}

At start of project to resolve the problems factor affecting on problems listed out.

\subsection{Variables/Parameters affecting on problems}

1) Input strip temperature

2) Process line speed

3) Passivation solution temperature

4) Passivation solution concentration

5) Hot air generator temperature

6) Temperature of strip before entering dryer

7) Temperature inside the dryer

8) Output temperature of strip

9) Moisture in dryer

\subsubsection{Role of variable}

1) Input strip temperature: Role of this parameter in occurring problems in passivation is important because temperature of input strip for perfect passivation should be in certain range. From observation we come two points. Point one is when strip temperature is below atmospheric temperature it carries water with it which is not evaporated in dryer and improper passivation applies on strip. Point two is when strip temperature in the range of $55-65^{\circ} \mathrm{C}$ powder formation is observed on strip in hexavalant chromic acid

2) Process line speed: Process line speed is depending on thickness of the input strip. High in thinner gauge strip and low in thicker gauge material.

3) Passiavtion solution temperature: As per supplier of the both solution passivation solution temperature should be in the range of $50-60^{\circ} \mathrm{C}$.

4) Passivation solution concentration: As per supplier of solution, for hexavalant chromic acid concentration range should be $15-20 \%$ by volume and for trivalent chromic acid concentration range should be $30-40 \%$ by volume

5) Hot air generator temperature: Hot air generator temperature is most important parameter on defect. At the start we are following the trial and error method for the drying the solution in dryer. Capacity of hot air dryer is $600^{\circ} \mathrm{C}$. We started from the temperature setting from $450^{\circ} \mathrm{C}$ for observation
6) Temperature of strip before entering dryer: This is temperature between the spraying unit of the solution dryer. This is depend on the solution temperature as well as input strip temperature

7) Temperature inside the dryer: This temperature is plays important role in the drying of solution. Dryer temperature is maintained through duct carrying hot air from hot air generator. We are observing the drop of temperature through duct.

8) Output temperature of the strip: This temperature is measured in between the dryer and deflector roll. Purpose of measuring strip temperature is to find out how to avoid the deposition of the solution on the roll. 9) Moisture in the dryer: Effect of the moisture is improper drying in both types of solution which cause deposition of solution on roll. We find out the sources of the moisture in dryer. From observation, moisture formation is when we start the passivation unit after long time or if in process breakdown occurs.

\section{Modification in passivation unit verses existence condition /proposed work}

From observation we comes to following modification required in exist Passivation unit i.e. work done in modification Passivation unit.

5.1 Heating Coil: Purpose of the heating coil to maintain the temperature of Passivation solution. Range of the Passivation solution temperature is 55$60^{\circ}$. So we are going to install electric heater in the solution tank.

5.2 HOT Air Caring Duct Design From Hag To Had: Purpose of the duct design is to avoid to temperature drop while carrying the hot air from HAG to HAD. We preferred the circular duct/pipe.

5.3 Inlet Blower: It has installed in two sections. First at HAG section and second is at before dryer. previously only one blower is there which is at HAG.

5.4 Exhaust Blower: Requirement of the exhaust blower to maintain the proper ventilation inside the dryer. This blower has installed at top of the dryer.

5.5 Dilution Fan: Purpose of the fan is to cool the strip before the deflector roll.

\section{Pareto Analysis of the observation of variables}

Pareto Analysis is a statistical technique in decisionmaking used for the selection of a limited number of tasks that produce significant overall effect. It uses the Pareto Principle (also known as the 80/20 rule) the idea that by doing $20 \%$ of the work you can generate $80 \%$ of the benefit of doing the entire job Pareto analysis made for find out which variable is more effective for the problems. 
In this we have done Pareto analysis of different variables for time and material diverted. This analysis is done for both passivation solutions. i.e. .hexavalent and trivalent. Following are the pareto analysis for both passivation solution from the readings taken when passivation is in running.

\subsection{First Pareto analysis}

\begin{tabular}{|c|c|c|c|c|}
\hline $\begin{array}{l}\frac{0}{\pi} \\
\frac{\pi}{\pi} \\
\stackrel{\pi}{\pi} \\
\frac{\pi}{3}\end{array}$ & 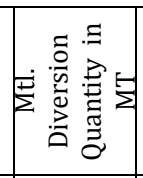 & 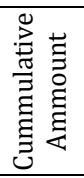 & 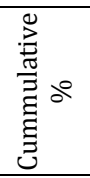 & 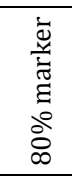 \\
\hline HAD Temp. & 25 & 25 & $63 \%$ & $80 \%$ \\
\hline Process Speed & 5 & 30 & $76 \%$ & $80 \%$ \\
\hline Moisture & 3 & 33 & $83 \%$ & $80 \%$ \\
\hline Exhaust Temp & 2 & 35 & $88 \%$ & $80 \%$ \\
\hline Incoming stip & \multirow{2}{*}{1.8} & \multirow{2}{*}{36.8} & \multirow{2}{*}{$93 \%$} & \multirow{2}{*}{$80 \%$} \\
\hline temp & & & & \\
\hline Solution Temp & 1.5 & 38.3 & $97 \%$ & $80 \%$ \\
\hline Outgoing strip & \multirow{2}{*}{1.3} & \multirow{2}{*}{39.6} & \multirow{2}{*}{$100 \%$} & \multirow{2}{*}{$80 \%$} \\
\hline Temp. & & & & \\
\hline
\end{tabular}

\begin{tabular}{|c|c|c|c|c|}
\hline $\begin{array}{l}\frac{0}{0} \\
\frac{\pi}{\pi} \\
\frac{\pi}{\pi} \\
\frac{\pi}{5}\end{array}$ & 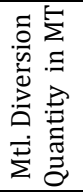 & 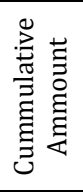 & 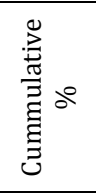 & 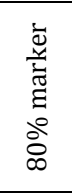 \\
\hline HAD Temp. & 25 & 25 & $63 \%$ & $80 \%$ \\
\hline Process Speed & 5 & 30 & $76 \%$ & $80 \%$ \\
\hline Moisture & 3 & 33 & $83 \%$ & $80 \%$ \\
\hline Exhaust Temp & 2 & 35 & $88 \%$ & $80 \%$ \\
\hline Incoming stip & \multirow{2}{*}{1.8} & \multirow{2}{*}{36.8} & \multirow{2}{*}{$93 \%$} & \multirow{2}{*}{$80 \%$} \\
\hline temp & & & & \\
\hline Solution Temp & 1.5 & 38.3 & $97 \%$ & $80 \%$ \\
\hline Outgoing strip & \multirow{2}{*}{1.3} & \multirow{2}{*}{39.6} & \multirow{2}{*}{$100 \%$} & \multirow{2}{*}{$80 \%$} \\
\hline Temp. & & & & \\
\hline
\end{tabular}

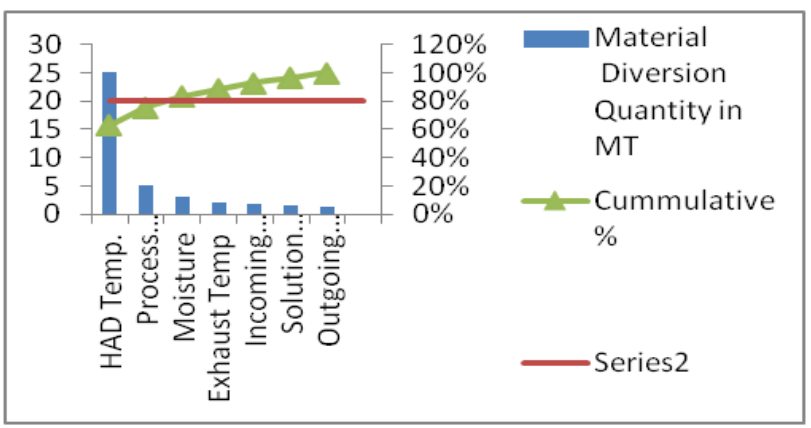

Chart1. Pareto chart for variable

\subsection{Second Pareto analysis}

This Pareto analysis is done to find out the operating temperature range for trivalent chromic acid.

\begin{tabular}{|c|c|c|c|c|}
\hline $\begin{array}{c}\text { Temp. in } \\
\text { Degrees }\end{array}$ & $\begin{array}{c}\text { Time } \\
\text { in } \\
\text { Min. }\end{array}$ & $\begin{array}{c}\text { cumulative } \\
\text { Time }\end{array}$ & $\begin{array}{c}\text { cumulative } \\
\%\end{array}$ & $80 \%$ \\
\hline Temp.150 & 420 & 420 & $10 \%$ & $80 \%$ \\
\hline Temp.160 & 420 & 840 & $21 \%$ & $80 \%$ \\
\hline Temp.165 & 420 & 1260 & $31 \%$ & $80 \%$ \\
\hline Temp.170 & 420 & 1680 & $42 \%$ & $80 \%$ \\
\hline Temp.145 & 410 & 2090 & $52 \%$ & $80 \%$ \\
\hline Temp.140 & 400 & 2490 & $62 \%$ & $80 \%$ \\
\hline Temp.135 & 360 & 2850 & $71 \%$ & $80 \%$ \\
\hline Temp.130 & 300 & 3150 & $79 \%$ & $80 \%$ \\
\hline Temp.125 & 240 & 3390 & $85 \%$ & $80 \%$ \\
\hline Temp.110 & 230 & 3620 & $90 \%$ & $80 \%$ \\
\hline Temp.100 & 210 & 3830 & $96 \%$ & $80 \%$ \\
\hline Temp.90 & 180 & 4010 & $100 \%$ & $80 \%$ \\
\hline & 4010 & \multicolumn{4}{l}{}
\end{tabular}

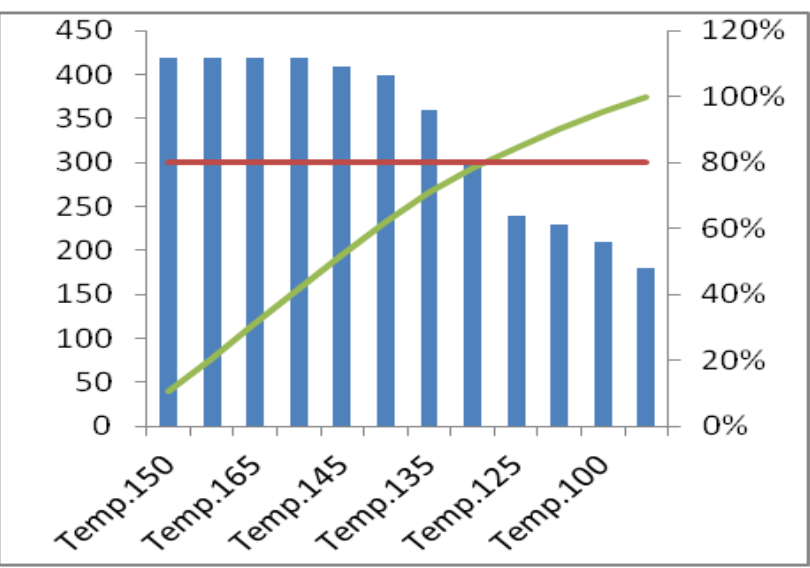

Chart 2 Pareto chart for operating temp. range of trivalent chromic solution

\subsection{Third Pareto analysis}

This Pareto analysis is done to find out the operating temperature range for hexavalent chromic acid.

\begin{tabular}{|c|c|c|c|c|}
\hline $\begin{array}{c}\text { Temp. in } \\
\text { Degrees }\end{array}$ & $\begin{array}{c}\text { Time } \\
\text { in } \\
\text { Min. }\end{array}$ & $\begin{array}{c}\text { Cumulative } \\
\text { time }\end{array}$ & $\begin{array}{c}\text { Cumulative } \\
\%\end{array}$ & $\begin{array}{c}80 \% \\
\text { marker }\end{array}$ \\
\hline Temp.140 & 480 & 480 & $11 \%$ & $80 \%$ \\
\hline Temp.135 & 460 & 940 & $22 \%$ & $80 \%$ \\
\hline Temp.130 & 450 & 1390 & $33 \%$ & $80 \%$ \\
\hline Temp.125 & 440 & 1830 & $43 \%$ & $80 \%$ \\
\hline Temp.120 & 430 & 2260 & $53 \%$ & $80 \%$ \\
\hline Temp.115 & 400 & 2660 & $63 \%$ & $80 \%$ \\
\hline Temp.110 & 350 & 3010 & $71 \%$ & $80 \%$ \\
\hline Temp.105 & 320 & 3330 & $78 \%$ & $80 \%$ \\
\hline Temp.100 & 300 & 3630 & $85 \%$ & $80 \%$ \\
\hline Temp.95 & 230 & 3860 & $91 \%$ & $80 \%$ \\
\hline Temp.90 & 210 & 4070 & $96 \%$ & $80 \%$ \\
\hline Temp.85 & 180 & 4250 & $100 \%$ & $80 \%$ \\
\hline \multirow{nyyyy}{*}{450} & & & \\
\cline { 2 - 5 } & 4250 & & \\
\hline
\end{tabular}




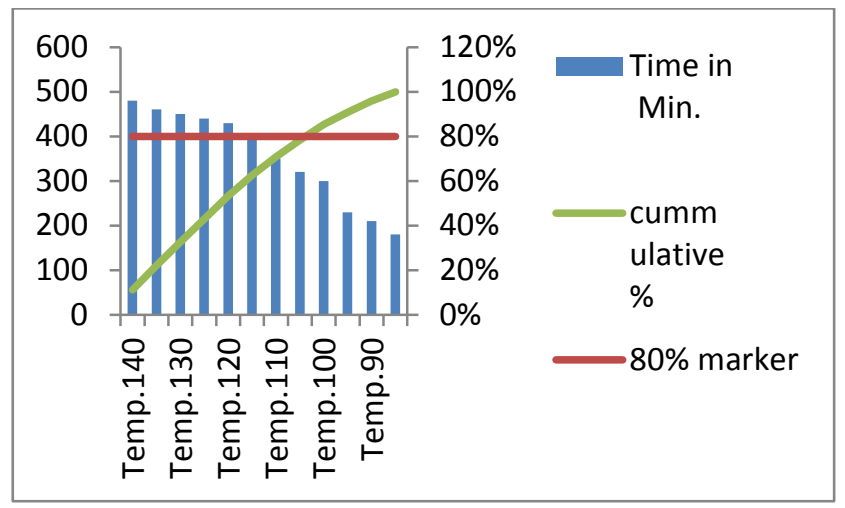

Chart 2. Pareto chart for operating temp. range of hexavalent chromic solution

\section{Conclusions}

From the Pareto analysis suitable temperature range for the different solution we get is

1) For chromic acid (cr+6) is $125-145^{\circ} \mathrm{c}$

2) For chromic acid (cr+3) is $135-165^{\circ} \mathrm{C}$

3) For chromic acid ( $\mathrm{cr}+6)$ concentration range is 10$15 \%$ by volume

4) For chromic solution $(\mathrm{cr}+3)$ concentration range is 28-35\% by volume

\section{Discussion \& benefits}

From the observations and analysis we have discussed following points for better performance of the passivation unit. We can say Standard Operating Procedure (SOP) for Passivation unit.

1) Start exhaust blower of Hot Air Dryer.

2) Stat Hot Air Supplying Blower.

3) Start Hot Air Generator and Set the temperature for respective solution

4) Open valve of hot air carrying duct after achieving temperature in HAG.

5) Take squeeze roll down and maintain the pressure $3 \mathrm{Kg} / \mathrm{cm} 2$

6) Start passivation solution spray pump.

7) Adjust the spray as per requirement on the running strip.

8) Maintain the constant process speed

\section{Benefits of the Modification}

1) Improvement in the surface quality of the product

2) Reduction in customer complaints

3) Increased productivity.

4) Saving in finished product loss

5) Increase in life of the solution.

6) Material will be processed as per the planning

7) OTIF will be maintained

8) Safe work environment

\section{References}

Hong Wang Characterization Of Dark Green Passivation Film On Galvanized Steel Sheet College of Mechanical and Electronic Engineering, Weifang University, Weifang, China Received: October 17, 2013

Passivation of Stainless Steel by by Dan Englebert, Vice President Technical Services Imagineering Enterprises, I

T.S.N. Sankara NarayananSurface Pretreatment By Phosphate Conversion Coatings A Review National Metallurgical Laboratory, Madras Centre CSIR, Complex, Taramani, Chennai600 113, India Received: April 22, 2005

Virendra V. Khakre, Avinash M. WankhadeReview paper on Design and Computational Analysis of Air Flow throuh Cooling Duct International Journal of Scientific \& Engineering Research, Volume 5, Issue 9, September-2014

M.Sampathkumar, Dsvsra Varaprasad, Vijaykumar, Static Analysis of Centrifugal Blower Using Composite Material The International Journal Of Engineering And Science (IJES), 2014, pp 2319 - 1805

H.Karbesian, A.E.Tekkaya A Review on hot stamping Institute of formation technology and lightweight construction,Journal of material processing technology, received oct.2010

White Rust Prevention An Industry Update and Guide Paper 2012 Presented By: Association of Water Technologies (AWT)

Thermal Sprayed Coatings Used Against Corrosion and Corrosive Wear

P. Fauchais and A. Vardelle SPCTS, UMR 7315, University of Limoges, France

Hot dip galvanizing data sheet published by galvanizing association wrens court,56 Victoria road, Sutton codified, west midlands.

Grace Chang, Charles Kerobo, MirjamHerrlich-Loos, Helmut Witteler RoHS Compliant, Non-Chrome Passivation of Galvanized Steel with LugalvanTMPassivation Products Product Development Surface Finishing

Hiroshi Kagechika** Managing Director, Dr., Steel Technology Centre Recent Progress and Future Trends in the Research and Development of Steel

Fan Performance By: Mark Stevens AMCA International Deputy Executive Director-Technical Affairs

Advanced Strategy Guideline: Air Distribution Basics and Duct Design Arlan Burdick IBACOS, Inc. December 2011. 